\title{
Lokiarchaeon exhibits homoacetogenesis
}

Authors: William D. Orsi ${ }^{1,2^{*}}$, Aurèle Vuillemin ${ }^{1}$, Paula Rodriguez ${ }^{1}$, Ömer K. Coskun ${ }^{1}$, Gonzalo V. Gomez-Saez ${ }^{3}$, Gaute Lavik ${ }^{5}$, Volker Morholz ${ }^{4}$, Timothy G. Ferdelman ${ }^{5}$

Affiliations:

1. Department of Earth and Environmental Sciences, Paleontology \& Geobiology, LudwigMaximilians-Universität München, 80333 Munich, Germany.

2. GeoBio-Center ${ }^{\mathrm{LMU}}$, Ludwig-Maximilians-Universität München, 80333 Munich, Germany

3. Research Group for Marine Geochemistry (ICBM-MPI Bridging Group), Institute for Chemistry and Biology of the Marine Environment, Carl von Ossietzky University of Oldenburg, D-26111, Oldenburg, Germany

4. Baltic Sea Research Institute, Warnemünde, Seestrasse 15, D18119 Rostock, Germany

5. Department of Biogeochemistry, Max Planck Institute for Marine Microbiology, Bremen, Germany

*To whom correspondence should be addressed: w.orsi@1rz.uni-muenchen.de

Keywords: Lokiarchaeota, Asgard archaea, acetogenesis, homoacetogenesis, eukaryogenesis

Abstract: The proposed Asgard superphylum of Archaea comprises the closest archaeal relatives of eukaryotes, whose genomes hold clues pertaining to the nature host cell that acquired the mitochondrion at the origin of eukaryotes ${ }^{1-4}$. Genomes of the Asgard candidate Phylum 'Candidatus Lokiarchaeota' [Lokiarchaeon] suggest an acetogenic $\mathrm{H}_{2}$-dependent lifestyle $^{5}$ and mixotrophic capabilities ${ }^{6}$. However, data on the activity of Lokiarchaeon are currently lacking, and the ecology of the host cell that acquired the mitochondrion is debated $^{4,7}$. Here, we show that in anoxic marine sediments underlying highly productive waters on the Namibian continental shelf Lokiarchaeon gene expression increases with depth below the seafloor, and was significantly different across a redox gradient spanning hypoxic to sulfidic conditions. Notably, Lokiarchaeon increased expression of genes involved in growth, carbohydrate metabolism, and the $\mathrm{H}_{2}$-dependent Wood-Ljungdahl (WLP) carbon fixation pathway under the most reducing (sulfidic) conditions. Quantitative stable isotope probing experiments revealed multiple populations of Lokiarchaeota utilizing both $\mathrm{CO}_{2}$ and diatomaceous extracellular polymeric substances (dEPS) as carbon sources over a 10-day incubation under anoxic conditions. This apparent anaerobic mixotrophic metabolism was consistent with the expression of Lokiarchaeon genes involved in transport and fermentation 
of sugars and amino acids. Remarkably, several Asgard populations were more enriched with ${ }^{13} \mathrm{C}$-dEPS compared to the community average, indicating a preference for dEPS as a growth substrate. The qSIP and gene expression data indicate a metabolism of "Candidatus Lokiarchaeota" similar to that of sugar fermenting homoacetogenic bacteria ${ }^{8}$, namely that Lokiarchaeon can couple fermentative $\mathrm{H}_{2}$ production from organic substrates with electron bifurcation and the autotrophic and $\mathrm{H}_{2}$-dependent WLP. Homoacetogenesis allows to access a wide range of substrates and relatively high ATP gain during acetogenic sugar fermentation ${ }^{8}$ thereby providing an ecological advantage for Lokiarchaeon in anoxic, energy limited settings.

The Benguela upwelling system is one of the most productive ecosystems of the world's oceans and exhibits an oxygen minimum zone (OMZ) overlying the seafloor on the Namibian continental shelf ${ }^{9}$. Below the Namibian seafloor relatively high amounts of sulfide $\left(\mathrm{H}_{2} \mathrm{~S}\right)$ and $\mathrm{H}_{2}$ are produced by sulfate reduction and microbial fermentation, respectively ${ }^{10}$. During the Southern Winter (July) of 2018 we sampled a $30 \mathrm{~cm}$-long sediment core underlying hypoxic bottom waters of the Namibian OMZ (Extended Data Fig. 1). The sediments exhibited a redox gradient spanning hypoxic (ca. $25 \mu \mathrm{M} \mathrm{O}_{2}$ ) conditions at the seafloor surface to sulfidic conditions at $30 \mathrm{~cm}$ below seafloor (cmbsf) (Fig. 1a).

Microbial abundance based on quantitative PCR (qPCR) of 16S rRNA genes showed that microbial biomass generally decreased with depth, with a subseafloor peak in biomass at the $\mathrm{NO}_{3}{ }^{-}$

- $\mathrm{H}_{2} \mathrm{~S}$ interface. High-throughput sequencing of the 16S rRNA genes showed the presence of diverse microbial communities, dominated by the Chloroflexi (Extended Data Fig. S2) and Proteobacteria. In contrast, Archaea represented on average only $1.9 \%$ (SD: $0.6 \%, \mathrm{n}=28$ samples) of the 16S rRNA gene sequences (Fig. 1c). Phylogenetics of the 16S rRNA genes showed that the

58 presence of Lokiarchaeota was attributed to two separate populations detected throughout the core 59 (Extended Data Fig. S3). To check whether PCR primers were biasing the relative abundance of 60 taxa, we performed direct (unamplified) sequencing of DNA extracted from three depths. 61 Metagenomic analysis of these samples showed that protein encoding open reading frames (ORFs) assigned to Deltaproteobacteria were by far the most abundant at all depths, and that Archaea were

$633.1 \%$ (SD: $1.1 \%, \mathrm{n}=3$ samples) of ORFs (Fig 1d). The relative abundance of "Candidatus 64 Lokiarchaeota" ORFs was much higher in the unamplified metagenomes (average 2.2\%, SD=0.9, $\mathrm{n}=3$ samples) compared to the relative abundance in 16S rRNA gene amplicon data (average 
0.01\%, SD: $0.004 \%, \mathrm{n}=28$ samples). The comparably lower abundance of Lokiarchaeon in the 16S rRNA gene sequences is likely due to PCR primer biases against the Asgard archaea. A relative abundance for "Candidatus Lokiarchaeota" (previously referred to as the "deep sea archaeal group") at ca. $2 \%$ in our metagenomes is still relatively low compared to the abundance of this group in other studies of anoxic marine sediments where it can reach up to $10-50 \%$ relative abundance deeper in the subsurface ${ }^{2,11}$.

After applying metatranscriptomics to the same samples (Extended Data Table S1), expressed ORFs with highest similarity to predicted proteins from archaeal genomes were on average $36 \%$ (SD: 7\%, $\mathrm{n}=8$ samples) of total ORFs detected (Fig. 1d). Of these, on average $50 \%$ (SD: 11\%, $\mathrm{n}=8$ samples) had highest similarity to 'Candidatus Lokiarchaeota', which increased significantly (Welch Two Sample t-test: $P=0.01$ ) up to $61 \%$ (SD: $0.2 \%, \mathrm{n}=8$ samples) in the deepest sample at 30 cmbsf (Fig. 1d). The relatively high amount of gene expression from Lokiarchaeon was inversely correlated with the abundance of Lokiarchaeon $16 \mathrm{~S}$ and metagenomic datasets. We normalized the group-specific gene expression against the relative abundance of those same groups in the metagenomes (see Methods). This analysis showed that Archaea (Lokiarchaeon and Bathyarchaeota) had higher levels of gene expression compared to all other bacterial groups - with the exception of Bacteroidetes at $30 \mathrm{cmbsf}$. Moreover, Lokiarchaeon-specific gene expression was overexpressed at all depths relative to metagenomes, and increased with depth below the seafloor being greatest in the sulfidic interval at $30 \mathrm{cmbsf}$ (Fig 2a). Genes with higher expression tended to have consistency between replicates, whereas those genes with lower expression have lower consistency between replicates, because the abundance of those transcripts are closer to our detection limit (Fig 2b). Moreover, across the entire redox spectrum sampled, gene expression of Lokiarchaeon was statistically significant (ANOSIM: $P=0.002, \mathrm{R}=0.76$ ) between the core top (hypoxic), $12 \mathrm{cmbsf}$ (nitrate/sulfide interface), and $28 \mathrm{~cm}$ (sulfidic) (Fig. 2b). This pattern of gene expression is strongly suggestive of an anaerobic metabolism for Lokiarchaeon.

The incomplete nature of Asgard archaea genomes together with the lower representation of 92 sequenced archaeal genomes compared to Bacteria ${ }^{12}$, make it likely that the high levels of archaeal 93 gene expression seen here are actually an underestimate. Indeed, after updating our database with

94 recently sequenced Lokiarchaeota $\mathrm{MAGs}^{13,14}$, the number of expressed ORFs in 95 metatranscriptomes with highest similarity to Lokiarchaeota MAGs increased nearly two fold 96 (from 522 to 982 ORFs). Lokiarchaeal MAGs comprise sequences from closely related strains 
97 and do not exhibit evidence of significant contamination ${ }^{15}$, thus we interpret expressed genes in 98 our metatranscriptomes with highest similarity to Lokiarchaeon MAGs as being derived from the 99 Lokiarchaeon group. However, we acknowledge that some genes in databases annotated as being 100 present in Asgard archaea MAGs might not actually derive from asgard archaeal chromosomes ${ }^{16}$, 101 but are present in environments where archaea occur and have been assigned to bins according to 102 criteria that differ from study to study.

103 Expression of ORFs with similarity to those encoding the anaerobic $\mathrm{H}_{2}$-dependent WLP by 104 Lokiarchaeon ${ }^{5}$ was observed predominantly in the sulfidic sediments (Fig 2b; CDS/ACS, mer, $105 \mathrm{mtd}$, fwdB, ftr, fdh). Since Lokiarchaeon lacks the key gene methyl-CoM reductase for 106 methanogenesis, the WLP likely functions as acetogenic (rather than methanogenic) ${ }^{5}$. 107 Furthermore, ORFs with similarity to heterodisulfide reductases (HdrABC) involved in hydrogen 108 based electron bifurcation ${ }^{17}$, and the coenzyme F420 NiFe hydrogenase (fhrB) ${ }^{5}$, were expressed 109 primarily in the deepest interval sampled at $30 \mathrm{cmbsf}$ (Fig. 2b). In methanogenic archaea, 110 hydrogen dependent electron bifurcation uses the MvhADG-HdrABC system ${ }^{17}$ but we did not 111 detect expression of MvhADG. Thus, it is possible that HdrABC based electron bifurcation in 112 Lokiarchaeon proceeds in a different manner, for example Desulfovibrio vulgaris uses FlxABCD$113 \mathrm{HdrABC}^{18}$. Autotrophs that fix $\mathrm{CO}_{2}$ via the WLP use pyruvate synthase (pyruvate:ferredoxin 114 oxidoreductase, por) to generate pyruvate ${ }^{5}$ and Lokiarchaeon expression of this archaeal por was 115 detected only in the interface and deepest (sulfidic) samples (Fig. 2b). These genes are typical of $116 \mathrm{H}_{2}$-dependent anaerobic autotrophs ${ }^{5}$, and their higher expression in the deepest (sulfidic) samples 117 indicates a stimulation of hydrogen metabolism and the acetogenic WLP.

118 Since $\mathrm{H}_{2}$ production from fermentation ${ }^{19}$ is common in aquatic sediment environments, and 119 high rates of sulfate reduction as an anaerobic terminal electron acceptor process have been 120 measured in surface sediments throughout the Namibian shelf ${ }^{20}$, it is reasonable to assume that in 121 the anoxic (sulfidic) depths of the sediment core sampled in our study that $\mathrm{H}_{2}$ production and 122 consumption is ongoing because this is a general feature of these environments ${ }^{21} . \mathrm{H}_{2}$ in anoxic 123 sediments is produced by fermentation and tightly coupled and controlled by hydrogenotrophic 124 bacteria and archaea using sulfate and bicarbonate as terminal electron acceptors ${ }^{21,10}$, but temporal 125 disturbances (e.g., bioturbation, variability in overlying water mass oxidant composition, or 126 variability in availability of carbon substrates) can lead to imbalances in $\mathrm{H}_{2}$ production and 127 consumption rates. For instance, non-equilibrium $\mathrm{H}_{2}$ values $>30 \mathrm{nmol} / \mathrm{L}$ have been observed in 
128 organic-rich continental slope sediments off Namibia ${ }^{10}$. Metabolic flexibility, such as possession

129 of the WLP and capacity for utilization of organic substrates for fermentation may allow

130 homoacetogenic Lokiarchaeon to take advantage of fluctuating $\mathrm{H}_{2}$ concentrations. Indeed, most

131 probable number and ${ }^{14} \mathrm{C}$ labeling experiments in similar sediments on the Chilean shelf suggested

132 that variable redox cycling provided ideal conditions for homoacetogenic microorganisms ${ }^{22}$.

133 In addition to expression of the WLP, Lokiarchaeota expressed genes involved in the transport

134 and metabolism of organic substrates, namely sugars and amino acids (Fig 2b). Glycolysis in

135 Lokiarchaeon was evidenced by expression of ORFs with similarity to fructose-bisphosate 136 aldolase and glyceraldehyde-3-phosphate dehydrogenase (Fig 2b: COG category G). Sugar 137 fermentation by Lokiarchaeon is evidenced by the expression of ORFs with similarity to pyruvate138 formate lyase that was expressed in the deepest samples (Fig 2b), which helps to regulate anaerobic 139 glucose metabolism by catalyzing the reversible conversion of pyruvate and coenzyme-A into 140 formate and acetyl-CoA ${ }^{23}$. Moreover, Lokiarchaeon expressed ORFs with similarity to an archaeal 141 fructose-1,6-bisphosphate aldolase (Fig 2b: COG category G), which is induced during sugar 142 fermentation in archaea and is responsible for sugar catabolism ${ }^{24}$.

143 Lokiarchaeon transcripts encoding amino acid and peptide transporters (Fig. 2b) further 144 indicate mixotrophic utilization of detrital proteins. The expression of acryloyl-CoA reductase 145 complex by Lokiarchaeon indicates that some of the amino acids are fermented, since this enzyme 146 is used for L-alanine fermentation ${ }^{25}$. Amino acid fermentations would provide Lokiarchaeon with 147 roughly one ATP per amino acid fermented ${ }^{4}$. This is consistent with amino acid fermentation ${ }^{14,26,27}$ 148 and mixotrophy ${ }^{28}$ as a general metabolic features of anaerobic archaea in anoxic marine sediments. 149 As these gene expression data suggested that Lokiarchaeota are anaerobic mixotrophic acetogens, 150 or homoacetogens ${ }^{8}$, we tested their ability to use ${ }^{13} \mathrm{C}$-labeled organic matter and ${ }^{13} \mathrm{CO}_{2}$ under 151 anoxic conditions.

152 In the deepest (sulfidic) sample, where Lokiarchaeon gene expression was highest (Figs. 1, 2) 153 we performed two sets of quantitative stable isotope probing (qSIP) experiments using ${ }^{13} \mathrm{C}$-labeled 154 bicarbonate and ${ }^{13} \mathrm{C}$-labeled dEPS (see Methods) to identify autotrophic and heterotrophic activity, 155 respectively. We used dEPS as a ${ }^{13} \mathrm{C}$-substrate for heterotrophic microbes because diatom derived 156 organic matter substantially contributes to the total organic matter in Namibian shelf sediments ${ }^{29}$. 157 The number of Asgard OTUs detected increased three fold at the end of the 10 day anoxic 158 incubations compared to the in situ (frozen) samples, indicating that Asgard archaea were enriched 
159 for under the incubation conditions. For example, two Lokiarchaeon OTUs were detected in the 160 frozen samples (Extended Data S3) whereas six Lokiarchaeon OTUs were detected at the end of 161 the incubation (Fig. 3c). The extent of ${ }^{13} \mathrm{C}$ enrichment in Asgard archaea populations from dEPS 162 was high compared Bacteria, and nearly all Asgard populations were also labeled in the ${ }^{13} \mathrm{C}$ 163 bicarbonate incubations suggesting mixotrophic activity (Fig. 3a). All Bathyarchaeota OTUs were 164 significantly labeled in the ${ }^{13} \mathrm{C}$-bicarbonate incubations, and to a lesser extent in the ${ }^{13} \mathrm{C}$-labeled 165 dEPS incubations compared to the Asgard populations (Fig. 3a). This experimental evidence 166 supports previous SIP studies showing that Bathyarchaeota utilize organic matter marine 167 sediments ${ }^{30,31}$, and the mixotrophic activity seen in our qSIP data supports genomic studies 168 suggesting that Bathyarchaeota are organo-heterotrophic acetogens ${ }^{32-34}$. The high ${ }^{13} \mathrm{C}$-enrichment 169 of the Lokiarchaeon and the Bathyarchaeota populations in the dEPS incubations, relative to the 170 bacteria, also supports the significantly higher gene expression levels of these groups in the deepest 171 sediment intervals (Fig. 2a).

172 All OTUs affiliated with "Candidatus Lokiarchaeota" $(\mathrm{n}=6)$ were significantly enriched with $173{ }^{13} \mathrm{C}$ in the bicarbonate incubations ( $\left.\mathrm{CI}>90 \%\right)$, with 5-35\% total carbon atoms per Lokiarchaeon 174 population being ${ }^{13} \mathrm{C}$-labeled (Fig. 3b). Five of the six Lokiarchaeon populations also exhibited a $175{ }^{13} \mathrm{C}$-labeling pattern that was suggestive of mixotrophic activity, as they had similar labeling in 176 both the bicarbonate and dEPS incubations (Fig. 3b). In comparison, an equal number of OTUs 177 affiliated with the "Candidatus Odinarchaeota" exhibited ${ }^{13} \mathrm{C}$-labeling patterns consistent with 178 mixotrophic and autotrophic activity, respectively (Fig. 3b). Three of these "Candidatus 179 Odinarchaeota" OTUs exhibiting autotrophy formed a separate, bootstrap supported clade together 180 with sequences from subseafloor and lake sediments (Fig. 3c). All detectable populations of 181 "Candidatus Heimdallarchaeota" exhibited ${ }^{13} \mathrm{C}$-labeling patterns consistent with a mixotrophic 182 metabolism (Fig. 3b, c). These results provide quantitative experimental support for genomic 183 studies indicating that anaerobic mixotrophy is widespread in the Asgard archaea ${ }^{6}$ and show that 184 some, particularly four populations affiliated with the "Candidatus Odinarchaeota" and one with 185 Lokiarchaeon (Fig. 3b), may obtain the majority of their carbon from autotrophy. There is always 186 a potential for cross-feeding in SIP experiments, whereby heterotrophs could feed off of the 187 necromass of more active chemolithoautotrophs. Since the majority of OTUs in the ${ }^{13} \mathrm{CO}_{2}$ 188 incubations were identified as significantly incorporating the label (Fig S4), it is indeed possible 189 that some cross feeding occurred over the 10 days in the ${ }^{13} \mathrm{CO}_{2}$ incubation. However, it would be 
190 very difficult to explain the expression of the $\mathrm{CO}_{2}$ fixation WLP pathway by Lokiarchaeon (Fig. $1912 \mathrm{~b}$ ), and the ${ }^{13} \mathrm{C}$-labeling pattern of Lokiarchaeon, if Lokiarchaeon was only getting labeled in the $192{ }^{13} \mathrm{CO}_{2}$ incubations by cross-feeding from more active autotrophic microbes.

193 The degree of ${ }^{13} \mathrm{C}$ enrichment of Lokiarchaeon populations in the ${ }^{13} \mathrm{C}$-labeled dEPS 194 incubations was higher than the bacterial average (Fig. 3a), providing quantitative evidence for 195 their important role in the degradation of diatom necromass and dEPS in anoxic sediments. 196 Clearly, the organic matter rich sediments ${ }^{29,35}$ on the Namibian shelf that we sampled represent an 197 environment that promotes a high activity of Lokiarchaeon. Since polysaccharides are the main 198 constituent of $\mathrm{dEPS}^{36}$ and we added this as a mixture of particulate and high molecular weight 199 dissolved organic matter (HMW-DOM), Lokiarchaeon is apparently well suited to access 200 polysaccharides from particulate and HMW-DOM. Mechanisms used by Lokiarchaeon to access 201 this organic matter are evident in the metatranscriptomes via expression of ORFs with similarity 202 to alpha-mannosidase (glycoside hydrolase family 38), pectate lyase (polysaccharide lyase family 203 9), cellobiose phosphorylase (glycoside hydrolase family 94) (Fig 2b: COG category G) that are 204 all involved in the degradation of complex carbohydrates ${ }^{37}$. These enzymes help to degrade 205 cellulose, pectin, and glycoproteins ${ }^{37}$ all of which are components of $\mathrm{dEPS}^{38}$. This heterotrophic 206 activity of "Candidatus Lokiarchaeota" (previously referred to as the DSAG group) is consistent 207 with a correlation of their abundance with organic matter in deeper subsurface sediment cores 208 down to 3.5 meters below the seafloor ${ }^{11}$.

209 A coupling of $\mathrm{H}_{2}$-dependent $\mathrm{CO}_{2}$ reduction with $\mathrm{H}_{2}$-producing fermentations is common in 210 homoacetogenic bacteria, that allows them to exploit otherwise-inaccessible organic substrates ${ }^{8}$. 211 Homoacetogens couple the WLP with a variety of organic electron donors (e.g., sugars, ethanol, 212 lactate), which is thought to be the most important ecological advantage of acetogens ${ }^{8}$. A good 213 example is acetogenic sugar fermentation performed during homoacetate fermentation 214 ("homoacetogenesis"), which provides the highest known ATP gain for glucose fermentations, 215 more than $4.3 \mathrm{ATP} / \mathrm{mol}$ glucose in $A$. woodii ${ }^{8}$. This relatively high ATP yield could explain the 216 high levels of anaerobic activity from "Candidatus Lokiarchaeota", that are exhibiting a similar 217 metabolism here, namely anaerobic utilization of carbohydrates, sugar fermentation, and the WLP. 218 Our data indicate that Lokiarchaeota exhibit homoacetogenesis, coupling $\mathrm{H}_{2}$-producing 219 fermentations with the $\mathrm{H}_{2}$-consuming WLP for $\mathrm{CO}_{2}$ fixation. This confirms earlier predictions for 
220 Lokiarchaeon metabolism based on metagenomics ${ }^{33}$ and adds to the growing evidence that 221 homoacetogenesis is a widespread metabolism in marine sediments ${ }^{34,39,40}$.

222 The relatively high activity and anaerobic mixotrophy of Asgard archaea in organic rich, 223 anoxic (sulfidic) Namibian shelf sediments, supports the genomic evidence that anaerobic 224 mixotrophic metabolism is widespread in the candidate Asgard superphylum ${ }^{4,6}$. The Asgard 225 archaea comprises the closest archaeal relatives of eukaryotes, and eukaryotes evolved during the 226 Proterozoic eon when ocean anoxia was widespread ${ }^{41,42} \cdot \mathrm{H}_{2}$ metabolism is furthermore 227 hypothesized as a feature of the host cell that acquired the mitochondrion ${ }^{43}$. It is thus conceivable 228 that $\mathrm{H}_{2}$-rich Proterozoic sediments under hypoxic waters with intense organic carbon flux to the 229 seafloor (features of our sampled environment), would have potentially promoted the activity of 230 LECA and represented an important habitat relevant to the emergence of the first eukaryotic cell. 
Figures.

233

A.

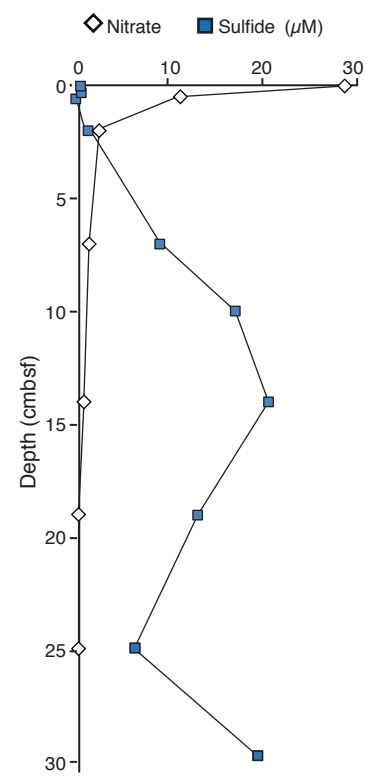

B.

$\mathrm{LOG}_{10}$ (16S rRNA gene copies $\mathrm{g}$ )

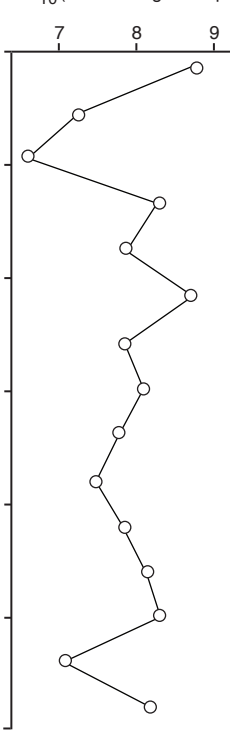

c.

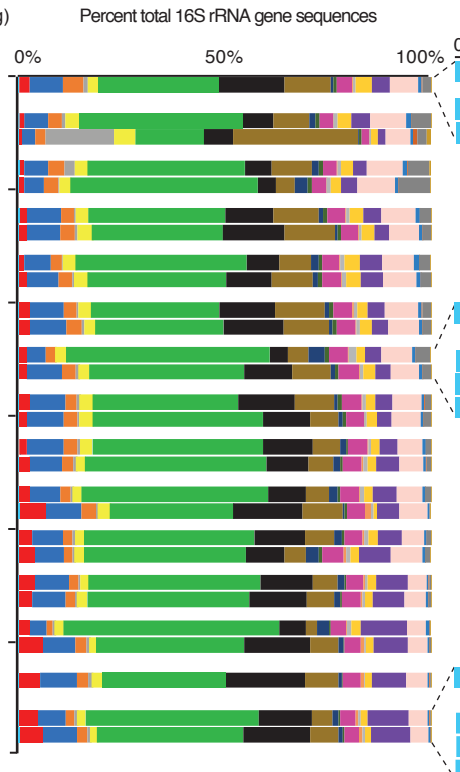

D.

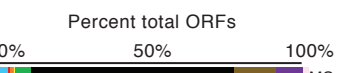

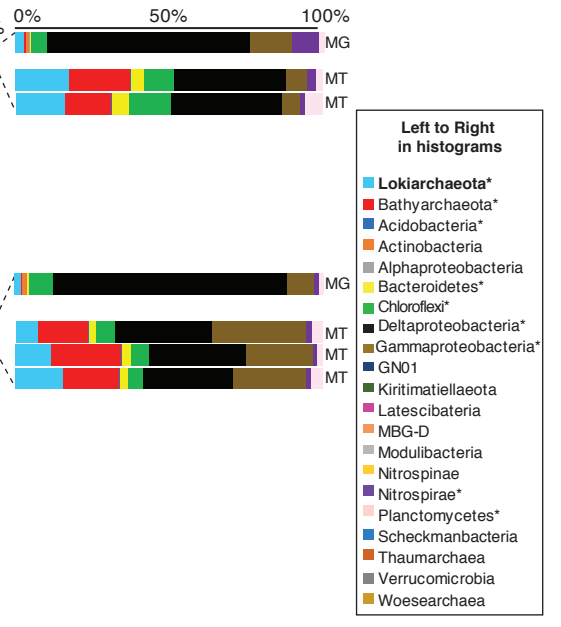

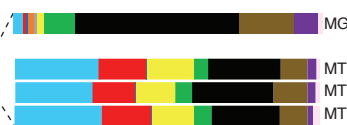

234

235

236

237

238

239

240

241

242

243

244

245

246

247

248

249

250

251

252

253

254

255

256

257

258
Figure 1: Lokiarchaeon gene expression increases in anoxic, sulfidic sediments. (A)

Geochemical profile of nitrate (diamonds) and sulfide (squares) showing a redox transition zone between 8 and $12 \mathrm{cmbsf}$. Sulfide data represent average measurements from two cores. (B) Abundance of total $16 \mathrm{~S}$ rRNA genes as determined by qPCR. (C) The relative abundance of $16 \mathrm{~S}$ rRNA gene sequences ( $\mathrm{n}=23,000$ average per sample, SD: 3,000) assigned to microbial groups, closely spaced histograms are biological replicates. (D) Relative abundance of ORFs in replicate metatranscriptomes (MT; $\mathrm{n}=1,069$ average per sample, SD: 238) with highest similarity to bacterial and archaeal groups. MG: Relative abundance of ORFs in unamplified metagenomes $(\mathrm{n}=57,526)$. Light blue $=$ Lokiarchaeota. Asterisks indicate groups detected in 16S, metatranscriptome, and metagenome datasets. 


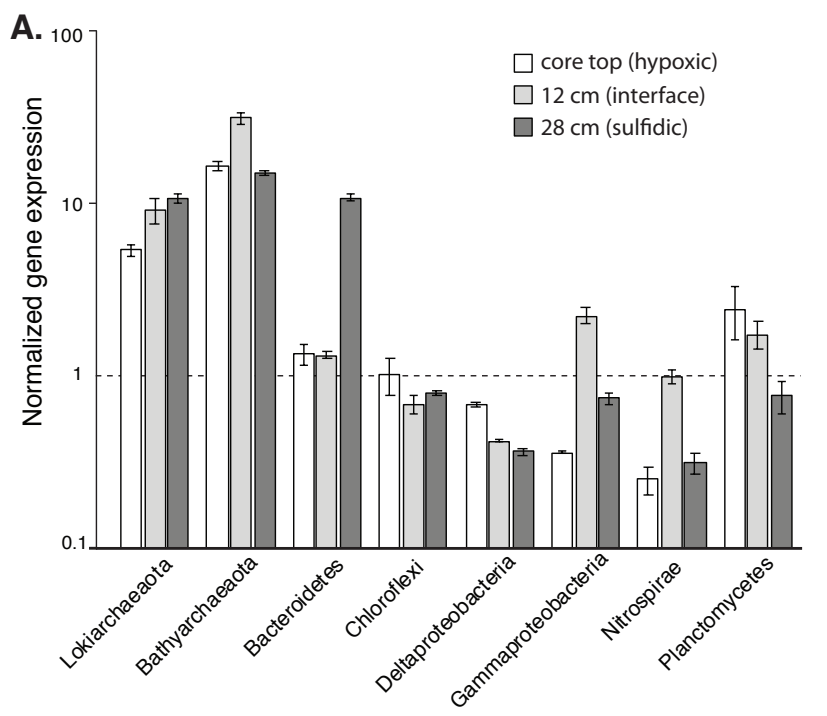

B.

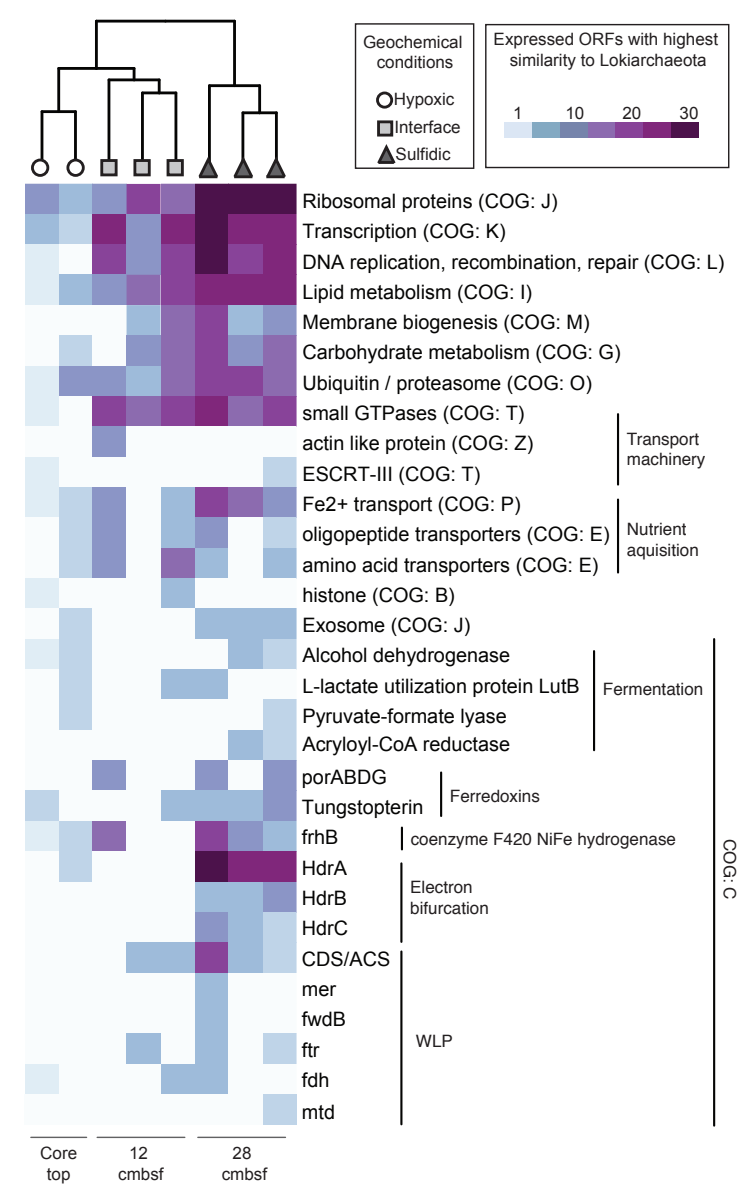

Figure 2: Lokiarchaeon gene expression across a hypoxic-sulfidic redox gradient.

(A) The y axis represents gene expression normalized against group abundance: $(\%$ total expressed ORFs per group / \% total ORFs per group in metagenomes). Error bars represent ranges in normalized values across biological replicates. Values above or below the dashed line are over- or underexpressed relative to the metagenomes, respectively.

(B) Heatmap showing the relative abundance of ORFs in metatranscriptomes with highest similarity to Lokiarchaeon MAGs ( $\mathrm{n}=982$ ORFs), columns represent biological replicates. The dendrogram is a UPGMA based on the differences in gene expression between the hypoxic, interface, and sulfidic depths. This was statistically significant (ANOSIM: $P=0.002, \mathrm{R}=0.76$ ). ORFs were classified into broad functional categories by comparison against the updated COG database $^{44}$. Genes involved in the putative $\mathrm{H}_{2}$-dependent carbon fixation pathway are classified according to Sousa $e t \mathrm{al}^{4}$. porABCD: 2-pyruvate:ferredoxin oxidoreductase, tungstopterin: Aldehyde ferredoxin oxidoreductase, $\mathrm{Hdr} A B C$ : heterodisulfide reductase, CDS/ACS: carbon monoxide dehydrogenase/acetyl-CoA synthase, mer: 5,10-methylene- $\mathrm{H}_{4}-$ methanopterin reductase, ftr: formyl transferase, fwdB: formyl-methanofuran dehydrogenase, fdh: formate dehydrogenase, mtd: 5,10-methylene- $\mathrm{H}_{4}$-methanopterin dehydrogenase. 


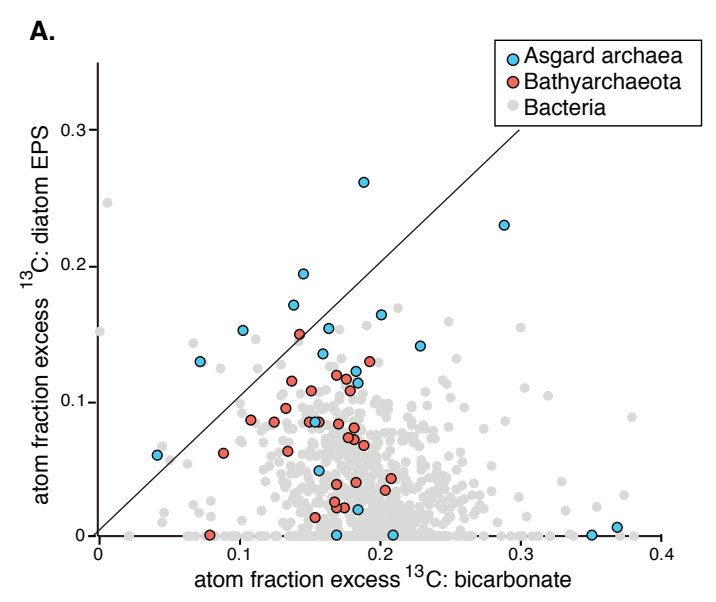

c.
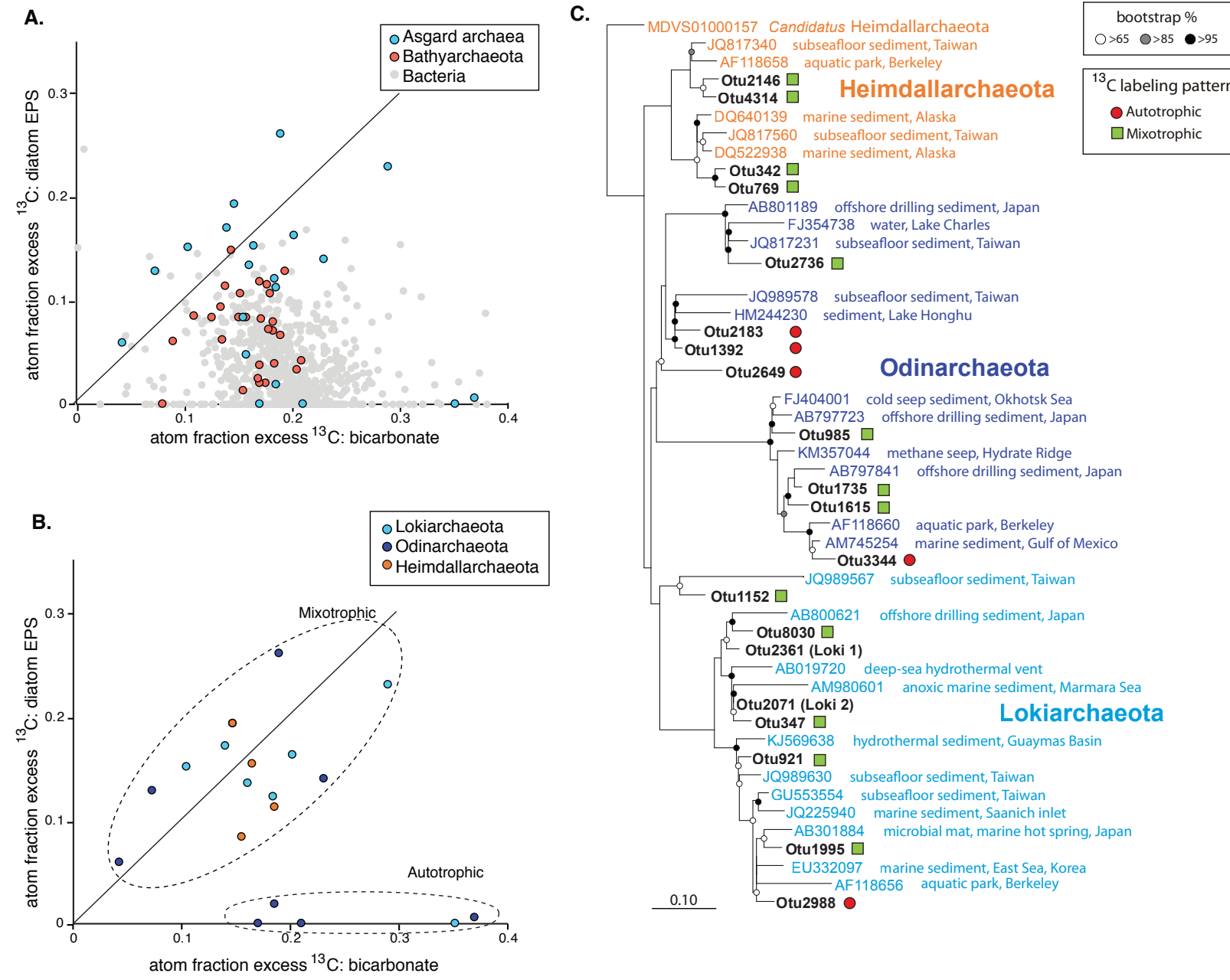

DQ522938 marine sediment, Alaska

- Otu342 $\square$

- AB801189 offshore drilling sediment, Japan

FJ354738 water, Lake Charles

- JQ817231 subseafloor sediment, Taiwan

Otu2736

- JQ989578 subseafloor sediment, Taiwan

- HM244230 sediment, Lake Honghu

Otu1392

Otu2649 O Odinarchaeota

FJ404001 cold seep sediment, Okhotsk Sea

- AB797723 offshore drilling sediment, Japan

- Otu985 $\square$

KM357044 methane seep, Hydrate Ridge

AB797841 offshore drilling sediment, Japan

- Otu1735

- Otu1615 $\square$

- AF118660 aquatic park Berkeley

AM745254 marine sediment, Gulf of Mexico Otu3344

Otu1152 $\square$

- AB800621 offshore drilling sediment, Japan

- Otu8030 $\square$

Otu2361 (Loki 1)

- AB019720 deep-sea hydrothermal vent

AM980601 anoxic marine sediment, Marmara Sea

Otu2071 (Loki 2)

Otu347 $\square$

- KJ569638 hydrothermal sediment, Guaymas Basin

Otu921 $\square$

JQ989630 subseafloor sediment, Taiwan

- GU553554 subseafloor sediment, Taiwan

JQ225940 marine sediment, Saanich inlet

AB301884 microbial mat, marine hot spring, Japan

Otu1995 $\square$

EU332097 marine sediment, East Sea, Korea

Otu2988 O

atom fraction excess ${ }^{13} \mathrm{C}$ : bicarbonate

Figure 3: qSIP shows high activity and mixotrophy by Asgard archaea. (A) Atom fraction ${ }^{13} \mathrm{C}$ with added ${ }^{13} \mathrm{C}$-bicarbonate and ${ }^{13} \mathrm{C}$-dEPS across all OTUs exhibiting significant labeling $(90 \% \mathrm{CI}$ not overlapping zero ${ }^{45}$ ) in at least one of the incubations. Each point represents the average atom fraction ${ }^{13} \mathrm{C}$ values for separate OTU across three biological replicates, axes units represent percent of ${ }^{13} \mathrm{C}$-labeled carbon atoms in $16 \mathrm{~S}$ rRNA genes per population in both incubations $(0.25$ indicates $25 \%$ of $\mathrm{C}$ atoms are ${ }^{13} \mathrm{C}$-labeled). (B) The same data, but displaying only Asgard archaea colored by candidate Phyla. The solid black lines indicate the expected relationship if organisms derived their carbon from bicarbonate and dEPS equally. The difference between the solid line and points falling above or below it is due to increased utilization of carbon from one of the other sources, and was used to define ${ }^{13} \mathrm{C}$-labeling patterns consistent with autotrophic and mixotrophic activity (dotted line circles) . (C) Phylogenetic tree of 16S rRNA genes from Asgard OTUs (from panel B) that exhibited significant ${ }^{13} \mathrm{C}$-labeling patterns. Loki1 and Loki2 OTUs are populations of Lokiarchaeon recovered from the pristine frozen core (Extended Data Figure 3). A complete summary of the qSIP results for all taxa is presented in Extended Data Figure 4. 
Acknowledgements. This work was supported by the Deutsche Forschungsgemeinschaft (DFG) through Project OR 417/4-1 (W.D.O), and the $F / S$ Meteor Expedition M148/2 'EreBUS'. The authors thank the captain and crew of the $F / S$ Meteor assistance during the oceanographic expedition, as well as S. Littmann, T. Wilkop, G. Klockgether and K. Imhoff who assisted in obtaining samples and providing chemical data. This work was performed in part through the Masters in Geobiology and Paleontology Program (MGAP) at LMU Munich. G.V.G-S was funded by the DFG project DI 842/6-1.

Author contributions. W.D.O, conceived the idea for the study and wrote the paper. T.G.F. organized and led the expedition. A.V., P.R., O.K.C, G.V.G-S., V.M., and T. G. F. produced data. W.D.O., A.V., P.R., G.L., and O.K.C, analyzed data. T.G.F., G.V.G-S., and W.D.O acquired the samples.

Competing interests. The authors declare no competing financial interests.

Additional information. Supplementary Information includes Extended Data Fig S1 - S4 and Table S1. All sequence data is publicly accessible in NCBI through BioProject number PRJNA525353.

Correspondence and requests for materials should be addressed to W.D.O.

\section{Methods:}

Sampling: A $30 \mathrm{~cm}$ long sediment core was obtained from a water depth of $125 \mathrm{~m}$ the Namibian continental shelf $(18.0 \mathrm{~S}, 11.3 \mathrm{E})$ during $F / S$ Meteor Expedition 'EreBUS' on July 10'th, 2018. Sediments were sampled with a multi corer (diameter $10 \mathrm{~cm}$ ), which yielded an intact sediment/water interface and the upper $30 \mathrm{~cm}$ of sediment. After retrieval, cores were moved immediately to a $4{ }^{\circ} \mathrm{C}$ cold room and sectioned every $2 \mathrm{~cm}$ within 24 hours. Sections were transferred immediately into sterile, DNA/RNA free $50 \mathrm{~mL}$ falcon tubes and then frozen immediately at $-20{ }^{\circ} \mathrm{C}$ until DNA and RNA extractions. For the determination of dissolved sulfide and nitrate in the sediments, pore waters were anaerobically extracted at $12^{\circ} \mathrm{C}$ from multi-cores M148-206-6-8 and M148-206-5-8 using Rhizon samplers inserted at 3 to $5 \mathrm{~cm}$ intervals through pre-drilled holes along the side of the core-barrels. Dissolved oxygen in the water column was measured using two Clark-type Seabird Electronics (SBE 43) oxygen sensors on a wireline Seabird Electronics (USA) CTD SBE 911+ with rosette water sampler. Sensor values were calibrated by Winkler determinations of dissolved oxygen on discrete samples ${ }^{43}$.

Pore water geochemistry: For sulfide analysis, $2 \mathrm{~mL}$ pore water aliquots were fixed with $0.5 \mathrm{~mL}$ of $5 \%(\mathrm{w} / \mathrm{w}) \mathrm{ZnCl}_{2}$ (Fisher Scientific). Total dissolved sulfide, which included $\mathrm{H}_{2} \mathrm{~S}_{\mathrm{aq}}+\mathrm{HS}^{-}+\mathrm{S}^{2+}$ and the sulfidic component of polysulfides $\mathrm{S}_{\mathrm{x}}{ }^{2-}$, was measured on board using the methylene blue method $^{46}$ on a Shimadzu UV120 Spectrophotometer. Sulfide was measured from two separate 30 
cm long cores collected via multi-coring. Nitrate was determined onboard with a QuAAtro39 autoanalyser (Seal Analytical) using the method based on Strickland and Parsons ${ }^{47}$.

$D N A$ and RNA extraction: DNA was isolated from $0.5 \mathrm{~g}$ sediment incubations aseptically in a sterile laminar flow hood using a sterile spatula, and extractions were carried out as described previously ${ }^{48}$. Purification of DNA extracts was carried out with the PowerClean Pro DNA Cleanup Kit (MO BIO Laboratories) and DNA was quantified with the Qubit dsDNA HS Assay kit (Thermo Fisher Scientific) according to manufacturer's instructions. DNA was extracted using a previously described protocol. In addition, DNA from laboratory dust and extraction blanks was extracted and purified in order to identify and remove any contaminant sequences introduced during the laboratory processing of the samples.

Total RNA was extracted from $0.5 \mathrm{~g}$ of sediment using the FastRNA Pro Soil-Direct Kit (MP Biomedicals) following the manufacturer's instructions with final elution of templates in $40 \mu \mathrm{L}$ PCR water (Roche) as described previously ${ }^{48}$ with some modifications to maximize RNA yield and reduce DNA contamination. The first modification was that, after the supernatant was removed after first homogenization step, a second homogenization was performed with an additional $500 \mu \mathrm{L}$ RNA Lysing Buffer. The tubes were centrifuged once again for 5 minutes at top speed, and the supernatant from the second homogenization was combined with that resulting from the first homogenization, continuing with the protocol from the manufacturer. Second, we added glycogen at a concentration of $1 \mu \mathrm{g} / \mathrm{mL}$ during the 30-minute isopropanol precipitation in order to maximize recovery of the RNA pellet. To reduce DNA contamination, we extracted all RNA samples in a HEPA-filtered laminar flow hood dedicated only for RNA work (no DNA allowed inside) that also contains dedicated RNA pipettors used exclusively inside the hood with RNA samples. All surfaces were treated with RNAse-Zap prior to extractions and exposed to UV light for 30 minutes before and after each extraction.

qPCR and 16S rRNA gene sequencing: Bacterial 16S rRNA gene V4 hypervariable region was performed with primer pair $515 \mathrm{~F} / 806 \mathrm{R}$ using the qPCR protocol described previously ${ }^{48}$. In brief, qPCR was carried out in $20 \mu \mathrm{L}$ solutions containing $10.4 \mu \mathrm{L}$ Sso Advanced SYBR green PCR buffer (Bio-Rad, Hercules, CA, USA), $0.4 \mu \mathrm{L}$ of $10 \mathrm{mM}$ primer, $6.8 \mu \mathrm{L}$ of nuclease-free water, and $2 \mu \mathrm{L}$ of the DNA template. Three technical replicates were prepared with the epMotion 5070 robotic pipetting system (Eppendorf), with a technical variation of $<5 \%$. All reactions were performed with a two-step protocol in a CFX Connect real-time PCR system (Bio-Rad, Hercules, CA, USA), including an enzyme activation step at $95^{\circ} \mathrm{C}$ for $3 \mathrm{~min}$, followed by 40 cycles of denaturation at $95^{\circ} \mathrm{C}$ for $15 \mathrm{~s}$ and then annealing at $55^{\circ} \mathrm{C}$ for $30 \mathrm{~s}$. qPCR standards consisted of 10 fold dilution series of the genes of interest that were PCR amplified from the sample using the same primers. Prior to the creation of the dilution series, the amplified standard was gel extracted and quantified with a Qubit instrument. The reaction efficiencies in all qPCR assays were between $90 \%$ and $110 \%$, with an $\mathrm{r}^{2}$ of 0.98 . Gene copies were normalized to the wet weight of the sediment.

Sequencing of the amplicons on the Illumina MiniSeq was performed as described previously ${ }^{48}$ through the LMU Munich GeoBio Center, which resulted in a total of 640,534 pairedend reads with an average of 23,000 reads per sample (SD: 3,000 reads) and USEARCH ${ }^{49}$ as described previously ${ }^{48}$. For $16 \mathrm{~S}$ rRNA genes, taxonomic assignments were generated by QIIME, version 1.9.1 ${ }^{50}$ using the implemented BLAST method against the SILVA rRNA gene database, release $132^{51}$. We removed all OTUs containing $<3$ sequences and which had no BLASTn hit. All OTUs that were found in the dust and extraction blank (contamination) samples were also 
removed from the dataset. Reads passing this quality control were then normalized by percentage of total sequencing depth per sample. Two OTUs were preliminarily assigned to Lokiarchaea based on the taxonomy provided in the BLASTn searches against SILVA ${ }^{51}$, and confirmed using phylogenetics (Fig. S2a).

Metatranscriptomics: DNAse treatment, synthesis of complementary DNA and library construction were obtained from $10 \mu \mathrm{L}$ of RNA templates by processing the Trio RNA-Seq kit protocol (NuGEN Technologies). Libraries were quantified on an Agilent 2100 Bioanalyzer System, using the High Sensitivity DNA reagents and DNA chips (Agilent Genomics). The libraries constructed using specific (different) barcodes, pooled at $1 \mathrm{nM}$, and sequenced in two separate sequencing runs with a paired-end 300 mid output kit on the Illumina MiniSeq. A total of 40 million sequences were obtained after Illumina sequencing, which could be assembled de novo into 41,230 contigs. Quality control, de novo assembly, and ORFs searches were performed as described previously ${ }^{52}$. A total of 8,556 ORFs were found that were then searched for similarity using BLASTp against a database containing predicted proteins from all fungal, bacterial, and archaeal genomes and MAGs in the JGI and NCBI databases using DIAMOND ${ }^{53}$. This database included all Asgard and Lokiarchaetoa MAGs from recent studies ${ }^{13,14}$, corresponding to 61,913 predicted proteins from Lokiarchaeota (as of July $26^{\text {th }}, 2019$ ). It also contained all ORFs assigned as Lokiarchaeota from the metagenomes prepared from the same samples, as well as all ORFs from the $>700$ transcriptomes of microbial eukaryotes from the MMETS project ${ }^{54}$. Cutoff for assigning hits to specific taxa were a minimum bit score of 50, minimum amino acid similarity of 30 , and an alignment length of 50 residues. Extraction blanks were also sequenced alongside the environmental samples to identify contamination, and ORFs from contaminant taxa. Contamination in the metatranscriptomes were primarily diatoms ("lab weeds"), cyanobacteria, Streptococcus, Acinetobacter, Staphylococcus, Rhizobium, Ralstonia, and Burkholderia. All ORFs that were shared between contaminant samples and the metatranscriptomes were removed prior to analysis. Incorporation of protist transcriptomes ${ }^{54}$ greatly reduced the amount of laboratory contamination from eukaryotic algae such as diatoms ("lab weeds") introduced during the library prep. All metatranscriptomes had $<10 \%$ ORFs from contaminating taxa.

Metagenomics: DNA was extracted according to the protocol described previously, which recovers ca. 10 times more DNA per gram sediment extracted compared to conventional protocols 55. The protocol yields more DNA because it involves a phosphate buffer that prevents DNA chelation to clay mineral surfaces, employs multiple freeze thaw steps, and concentrates the DNA with Amicon filters as opposed to precipitating with ethanol or isopropanol. This yielded microgram quantities of DNA from the core top, $12 \mathrm{~cm}$, and $28 \mathrm{~cm}$ samples $(1 \mathrm{~g}$ sediment was used for the extraction) and allowed us to create Illumina ready libraries from microgram quantities of extracted DNA without prior amplification. Illumina libraries were prepared, quality checked, and sequenced as described previously ${ }^{55}$. De novo assembly of raw reads passing quality control, ORF finding, and annotation were performed using the same pipeline applied previously for sediment metagenome datasets ${ }^{55}$ and the metatranscriptomes in this study (see above section). This database included all publicly available Asgards MAGs to date in the NCBI Protein database (corresponding to 61,913 predicted proteins from Lokiarchaeota as of July $26^{\text {th }}, 2019$ ). Extraction blanks were also sequenced alongside the environmental samples to identify contamination, and ORFs from contaminant taxa were removed prior to analysis. All metagenomes had $<1 \%$ ORFs from contaminating taxa. 
As an additional method for assessing the relative abundance of Lokiarchaeota in the metagenomes, raw reads from the were mapped against the assembled MAG of Lokiarchaeum sp. GC14_75, GenBank contig references no. JYIM010000001.1 to 505.1 (Spang et al., 2015) ${ }^{2}$ using Geneious v. 8.1.9. For this genome mapping, we ran 100 iterations at medium sensitivity, which entailed the following parameters: minimum mapping quality: $25 \%$, minimum coverage: 5 reads, strict threshold: $50 \%$. An average of $2 \%(+/-0.6 \%)$ reads mapped to the assembled Lokiarchaeota $\mathrm{MAG}^{2}$, which covered the entire genome (504/504 contigs recovered at all depths). This was comparable to the relative abundance of de novo assembled metagenome contigs encoding ORFs with highest similarity to Lokiarchaeota MAGs, which was $1.8 \%(+/-0.7 \%)$. Thus, both methods (genome recruitment vs. de novo assembly) indicate the same relative abundance for Lokiarchaeota at ca. $2 \%$ of the total community.

Normalizing gene expression: Group-specific gene expression levels were calculated as follows. The relative abundance of ORFs (defined as percent of total ORFs) with highest similarity to a particular group were normalized against the same relative abundance in the metagenome from the same sample. For this normalization, we only considered ORFs that had similarity to a database entry at relatively high confidence (see above). We chose to focus on total ORFs detected, as opposed to number of reads mapping per kilobase per ORF (e.g., RPKM), in order to reduce potential bias from small numbers of 'housekeeping' genes with potentially higher expression levels. Thus, by considering total number of ORFs expressed (as opposed RPKM values), the normalization displays the percentage of the pan-genome that is expressed as a function of group abundance. The normalized values are thus placed on a scale of either greater or less than 1 , corresponding to either over- or underexpression, respectively. In other words, if the relative abundance of expressed ORFs from a particular group is higher in the metatranscriptome relative to the metagenome, then the value will be above 1 . This calculation represents a group average in activity a semi-quantitative approach to compare the average level of transcriptional activity between Phylum level groups in the communities sampled.

Experimental set up for ${ }^{13} \mathrm{C}$ incubations: Sediments from a depth of $28 \mathrm{cmbsf}$ were incubated in triplicate for 10 days in the dark with either $2 \mathrm{mM} \mathrm{99 \%}{ }^{13} \mathrm{C}$-labeled or unlabeled (control) sodium bicarbonate $\left(\mathrm{NaHCO}_{3}\right.$; Sigma-Aldrich, St. Louis, $\left.\mathrm{MO}, \mathrm{USA}\right)$ at $10{ }^{\circ} \mathrm{C}$. A second set of incubations from the same sediment depth were set up with either ${ }^{13} \mathrm{C}$-labeled or unlabeled (control) diatom necromass / EPS, produced from a culture of the diatom Chaetocerous socialis (Norwegian Culture Collection strain K1676). C. socialis cells were grown in $250 \mathrm{~mL}$ sterile polystyrene culture flasks (VWR International) with L1 growth medium ${ }^{56}$ at $22{ }^{\circ} \mathrm{C}$ for seven days exposed to the natural light dark cycle (flasks were placed in an east-facing window). One set of cultures was grown with 2 $\mathrm{mM} 99 \%{ }^{13} \mathrm{C}$-labeled sodium bicarbonate and another set was grown with unlabeled sodium bicarbonate. After seven days the cultures were turbid, as evidenced by mucosal light brown flocculant (EPS and colonies of diatom cells) and the cultures at that point were concentrated in $50 \mathrm{KDa}$ Amicon filters. Thus, the resulting cell culture concentrates consisted primarily of particulate and high-molecular-weight dissolved organic matter. GC-IRMS was used to determine that the atom percent ${ }^{13} \mathrm{C}$ enrichment of the organic matter was $>50 \%$. For the incubations, flasks received either the unlabeled or ${ }^{13} \mathrm{C}$-labeled organic matter at a final concentration of $200 \mu \mathrm{g}$ per gram. As the total organic carbon content of sediments at a several nearby locations $(18.3 \mathrm{~S}, 11.5$ E) was determined previously to be between 0.5 and $2.3 \%{ }^{35}$, we added labeled organic matter at a 
concentration roughly equal to $1-3 \%$ of the in situ concentration. Since sinking diatom biomass is a major contributor to the organic carbon content of Namibian shelf sediments ${ }^{29}$, the ${ }^{13} \mathrm{C}$-labeled organic matter representing a mixture of dead diatom cells and their EPS serves as an appropriate proxy for tracking activity of heterotrophic microbes in the sediments.

Quantitative stable isotope probing: Sediment was added to $20 \mathrm{~mL}$ glass flasks leaving no headspace (ca. $20 \mathrm{~g}$ sediment) that were crimp sealed using grey butyl rubber stoppers. Anoxic conditions were confirmed by monitoring $\mathrm{O}_{2}$ concentrations non-invasively as described previously ${ }^{48}$ using a Fibox fiber optic $\mathrm{O}_{2}$ sensor. After 10 days the flasks were frozen until processing for SIP. DNA from the samples was extracted as described above and prepared for density gradient centrifugation according to the quantitative stable isotope probing protocol as described previously ${ }^{45}$. In brief, density gradient centrifugations were carried out in a TLN-100 Optima MAX-TL ultracentrifuge (Beckman Coulter, Brea, CA, USA) near-vertical rotor at $18{ }^{\circ} \mathrm{C}$ for $72 \mathrm{~h}$ at $165,000 \times \mathrm{g} .50 \mu \mathrm{L}$ of DNA spanning from $0.5 \mu \mathrm{g}$ to $1.5 \mu \mathrm{g}$ which was within the range of proposed values ${ }^{57}$ was added to a solution of cesium chloride $(\mathrm{CsCl})$ and gradient buffer $(0.1 \mathrm{M}$ Tris, $0.1 \mathrm{M} \mathrm{KCl}$ and $1 \mathrm{mM}$ EDTA) in order to achieve a starting density of $1.70 \mathrm{~g} \mathrm{~mL}^{-1}$ in a 3.3 mL polyallomer OptiSeal tubes (Beckman Coulter, Brea, CA, USA). After ultracentrifugation, the density gradients were fractionated into 15 equal fractions of $200 \mu \mathrm{L}$ from the bottom of polyallomer OptiSeal tubes by using a syringe pump and fraction recovery system (Beckman Coulter, Brea, CA, USA). The density of these fractions was measured with an AR200 digital refractometer (Reichert Analytical Instruments, Depew, NY, USA). DNA was precipitated from the fractions using 2 volumes of polyethylene glycol with $2 \mu \mathrm{L}\left(10 \mathrm{mg} \mathrm{mL}^{-1}\right)$ glycogen and precipitated overnight at room temperature. DNA was pelleted by centrifugation $(13,000 \times g ; 40$ min), washed with $70 \%$ ethanol, and resuspended with $30 \mu \mathrm{L}$ molecular-grade (DEPC-treated) water. DNA was quantified fluorometrically using a Qubit. The observed excess atom ${ }^{13} \mathrm{C}$ enrichment fraction (EAF) was calculated for each taxon according to a previously described study $^{45}$ using a qSIP workflow embedded in the HTS-SIP R package ${ }^{58}$. Weighted average densities were calculated for each taxon's DNA in the control $\left({ }^{12} \mathrm{C}\right.$ added $)$ and in the experimental incubation $\left({ }^{13} \mathrm{C}\right.$ added) as described Hungate et al. ${ }^{59}$ to estimate the atom fraction excess of ${ }^{13} \mathrm{C}$ for each OTU. To calculate the bootstrap confidence intervals (CI) for significant isotopic incorporation, bootstrap replicates $(n=1000)$ were run with the HTS-SIP R package ${ }^{58}$; an OTU was considered to be ${ }^{13} \mathrm{C}$ labeled if the $90 \%$ CI was above the $0 \%$ EAF cutoff ${ }^{59}$. 


\section{References:}

1 Eme, L., Spang, A., Lombard, J., Stairs, C. W. \& Ettema, T. J. G. Archaea and the origin of eukaryotes. Nat Rev Microbiol 15, 711-723 (2017).

2 Spang, A. et al. Complex archaea that bridge the gap between prokaryotes and eukaryotes. Nature 521, 173-179 (2015).

3 Zaremba-Niedzwiedzka, K. et al. Asgard archaea illuminate the origin of eukaryotic cellular complexity. Nature 541, 353-358 (2017).

4 Martin, W. F., Tielens, A. G. M., Mentel, M., Garg, S. G. \& Gould, S. V. The physiology of phagocytosis in the context of mitochondrial origin. Microbiol Mol Biol Rev 81, e0000800017 (2017).

5 Sousa, F. L., Neukirchen, S., Allen, J. F., Lane, N. \& Martin, W. F. Lokiarchaeon is hydrogen dependent. Nat Microbiol 1, 16034, doi:10.1038/nmicrobiol.2016.34 (2016).

6 Spang, A. et al. Proposal of the reverse flow model for the origin of the eukaryotic cell based on comparative analyses of Asgard archaeal metabolism. Nat Microbiol, (2019).

7 Lane, N. \& Martin, W. The energetics of genome complexity. Nature 467, 929-934, (2010).

8 Schuchmann, K. \& Müller, V. Energetics and application of heterotrophy in acetogenic bacteria. Applied and Environmental Microbiology 82, 4056-4069 (2016).

9 Lavik, G. et al. Detoxification of sulphidic African shelf waters by blooming chemolithotrophs. Nature 457, 581-584 (2009).

10 Lin, Y. S. et al. Towards constraining H2 concentrations in subseafloor sediment: A proposal for combined analysis by two distinct approaches. Geochimica et Cosmochimica Acta 77, 186-201 (2012).

11 Jorgensen, S. L. et al. Correlating microbial community profiles with geochemical data in highly stratified sediments from the Arctic Mid-Ocean Ridge. Proc Natl Acad Sci U S A 109, E2846-2855 (2012).

12 Spang, A., Caceres, E. F. \& Ettema, T. J. G. Genomic exploration of the diversity, ecology, and evolution of the archaeal domain of life. Science 357 (2017).

13 Seitz, K. W. et al. Asgard archaea capable of anaeribc hydrocarbon cycling. Nature Communications 10, 1822 (2019).

14 Dombrowski, N., Teske, A. P. \& Baker, B. J. Expansive microbial metabolic versatility and biodiversity in dynamic Guaymas Basin hydrothermal sediments. Nat Commun 9, 4999, (2018).

15 Spang, A. et al. Asgard archaea are the closest prokaryotic relatives of eukaryotes. PLoS Genet 14, e1007080 (2018).

16 Da Cunha, V., Gaia, M., Gadelle, D., Nasir, A. \& Forterre, P. Lokiarchaea are close relatives of Euryarchaeota, not bridging the gap between prokaryotes and eukaryotes. PLoS Genet 13, e1006810 (2017).

17 Buckel, W. \& Thauer, R. K. Energy conservation via electron bifurcating ferredoxin reduction and proton $/ \mathrm{Na}(+)$ translocating ferredoxin oxidation. Biochim Biophys Acta 1827, 94-113 (2013).

18 Ramos, A. R. et al. The FlxABCD-HdrABC proteins correspond to a novel NADH dehydrogenase/heterodisulfide reductase widespread in anaerobic bacteria and involved in ethanol metabolism in Desulfovibrio vulgaris Hildenborough. Environ Microbiol 17, 22882305 (2015). 
19 Lovley, D. R. \& Phillips, E. J. P. Hydrogen concentrations as an indicator of the predominant terminal electron-accepting reactions in aquatic sediments. Geochimica et Cosmochimica Acta 52, 2993-3003 (1988).

20 Schulz, H. D. et al. Dense populations of a giant sulfur bacterium in Namibian shelf sediments. Science 284, 493-495 (1999).

21 Schink, B. Energetics of syntrophic cooperation in methanogenic degradation. Microbiol Mol Biol Rev 61, 262-280 (1997).

22 Ferdelman, T. et al. Sulfate reduction and methanogenesis in a Thioploca-dominated sediment off the coast of Chile. Geochimica et Cosmochimica Acta 61, 3065-3079 (1997).

23 Becker, A. et al. Structure and mechanism of the glycyl radical enzyme pyruvate formatelyase. Nat Stuct Biol 6, 969-975 (1999).

24 Siebers, B. et al. Archaeal fructose-1,6-bisphosphate aldolases constitute a new family of archaeal type class I aldolase. J Biol Chem 276, 28710-28718 (2001).

25 Hetzel, M. et al. Acryloyl-CoA reductase from Clostridium propionicum. An enzyme complex of propionyl-CoA dehydrogenase and electron-transferring flavoprotein. Eur $J$ Biochem 270, 902-910 (2003).

26 Lloyd, K. G. et al. Predominant archaea in marine sediments degrade detrital proteins. Nature 496, 215-218 (2013).

27 Orsi, W. D. Ecology and evolution of seafloor and subseafloor microbial communities. Nat Rev Microbiol, doi:10.1038/s41579-018-0046-8 (2018).

28 Zhou, Z. et al. Genomic and transcriptomic insights into the ecology and metabolism of benthic archaeal cosmopolitan, Thermoprofundales (MBG-D archaea). ISME J 13, 885901 (2019).

29 Compton, J. S. \& Bergh, E. W. Phosphorite deposits on the Namibian shelf. Marine Geology 380, 290-314 (2016).

30 Seyler, L. M., McGuinness, L. M. \& Kerkhof, L. J. Crenarchaeal heterotrophy in salt marsh sediments. ISME J 8, 1534-1543 (2014).

$31 \mathrm{Yu}, \mathrm{T}$. et al. Growth of sedimentary Bathyarchaeota on lignin as an energy source. Proc Natl Acad Sci U S A 115, 6022-6027 (2018).

32 Lazar, C. S. et al. Genomic evidence for distinct carbon substrate preferences and ecological niches of Bathyarchaeota in estuarine sediments. Environ Microbiol 18, 12001211 (2016).

33 Martin, W. F., Neukirchen, S., Zimorski, V., Gould, S. B. \& Sousa, F. L. Energy for two: New archaeal lineages and the origin of mitochondria. Bioessays 38, 850-856 (2016).

34 He, Y. et al. Genomic and enzymatic evidence for acetogenesis among multiple lineages of the archaeal phylum Bathyarchaeota widespread in marine sediments. Nat Microbiol 1, 16035 (2016).

35 Inthorn, M., Wagner, T., Scheeder, G. \& Zabel, M. Lateral transport controls distribution, quality and burial of organic matter along continental slopes in high-productivity areas. Geology 34, 205-208 (2006).

36 More, T. T., Yadav, J. S., Yan, S., Tyagi, R. D. \& Surampalli, R. Y. Extracellular polymeric substances of bacteria and their potential environmental applications. J Environ Manage 144, 1-25 (2014).

37 Lombard, V., Golaconda Ramulu, H., Drula, E., Coutinho, P. M. \& Henrissat, B. The carbohydrate-active enzymes database (CAZy) in 2013. Nucleic Acids Res 42, D490-495, (2014). 
38 Aslam, S. N., Strauss, J., Thomas, D. N., Mock, T. \& Underwood, G. J. C. Identifying metabolic pathways for production of extracellular polymeric substances by the diatom Fragilariopsis cylindrus inhabiting sea ice. ISME J 12, 1237-1251 (2018).

39 Lever, M. A. Acetogenesis in the energy-starved deep biosphere - a paradox? Frontiers in Microbiology 2, 284 (2012).

40 Lever, M. A. et al. Acetogenesis in deep subseafloor sediments of the Juan de Fuca Ridge Flank: a synthesis of geochemical, thermodynamic, and gene-based evidence. Geomicrobiology Journal 27, 183-211 (2009).

41 Knoll, A. H. The early evolution of eukaryotes: a geological perspective. Science 256, 622627 (1992).

42 Lyons, T. W., Reinhard, C. T. \& Planavsky, N. J. The rise of oxygen in Earth's early ocean and atmosphere. Nature 506, 307-315 (2014).

43 Martin, W. \& Muller, M. The hydrogen hypothesis for the first eukaryote. Nature 392, 3741 (1998).

44 Galperin, M. Y., Makarova, K. S., Wolf, Y. I. \& Koonin, E. V. Expanded microbial genome coverage and improved protein family annotation in the COG database. Nucleic Acids Res 43, D261-269 (2015).

45 Coskun, O. K., Pichler, M., Vargas, S., Gilder, S. \& Orsi, W. D. Linking uncultivated microbial populations with benthic carbon turnover using quantitative stable isotope probing. Appl Environ Microbiol, doi:10.1128/AEM.01083-18 (2018).

46 Cline, J. D. Spectrophotometric determination of hydrogen sulfide in natural waters. Limnology and Oceanography 14, 454-458 (1969).

47 Strickland, J. D. \& Parsons, T. R. A practical handbook of seawater analysis. Vol. Bulletin 167 (Fisheries Research Board of Canada, 1972).

48 Ortega-Arbulu, A. S., Pichler, M., Vuillemin, A. \& Orsi, W. D. Effects of organic matter and low oxygen on the mycobenthos in a coastal lagoon. Environ Microbiol 21, 374-388, (2019).

49 Edgar, R. C. UPARSE: highly accurate OTU sequences from microbial amplicon reads. Nat Methods 10, 996-998 (2013).

50 Caporaso, J. G. et al. QIIME allows analysis of high-throughput community sequencing data. Nat Methods 7, 335-336 (2011).

51 Quast, C. et al. The SILVA ribosomal RNA gene database project: improved data processing and web-based tools. Nucleic Acids Res 41, D590-596 (2013).

52 Orsi, W. D., Richards, T. A. \& Francis, W. R. Predicted microbial secretomes and their target substrates in marine sediment. Nature Microbiology 3, 32-37 (2018).

53 Buchfink, B., Xie, C. \& Huson, D. H. Fast and sensitive protein alignment using DIAMOND. Nat Methods 12, 59-60 (2015).

54 Keeling, P. J. et al. The Marine Microbial Eukaryote Transcriptome Sequencing Project (MMETSP): illuminating the functional diversity of eukaryotic life in the oceans through transcriptome sequencing. PLoS Biol 12, e1001889 (2014).

55 Vuillemin, A. et al. Archaea dominate oxic subseafloor communities over multimillionyear time scales. Sci Adv 5, eaaw4108 (2019).

56 Guillard, R. R. L. \& Hargraves, P. E. Stichochrysis immobilis is a diatom, not a chrysophyte. Phycologia 32 (1993).

57 Dunford, E. A. \& Neufeld, J. D. DNA stable-isotope probing (DNA-SIP). J. Vis. Exp., (2010). 
58 Youngblut, N. D., Barnett, S. E. \& Buckley, D. H. HTSSIP: An R package for analysis of high throughput sequencing data from nucleic acid stable isotope probing (SIP) experiments. PLoS One 13, e0189616 (2018).

59 Hungate, B. A. et al. Quantitative microbial ecology through stable isotope probing. Appl. Environ. Microbiol. 81, 7570-7581 (2015). 